\title{
Coherent synthesis of ultra-broadband optical parametric amplifiers
}

\author{
C. Manzoni ${ }^{1}$, S.W. Huang ${ }^{2}$, G. Cirmi ${ }^{2}$, J. Moses ${ }^{2}$, F. X. Kärtner ${ }^{2,3}$, and G. Cerullo ${ }^{1}$ \\ ${ }^{I}$ IFN-CNR, Dipartimento di Fisica, Politecnico di Milano, Piazza L. Da Vinci 32, 20133 Milano, Italy \\ ${ }^{2}$ Department of Electrical Engineering and Computer Science and Research Laboratory of Electronics, \\ Massachusetts Institute of Technology, Cambridge, Massachusetts 02139, USA \\ ${ }^{3}$ Center for Free-Electron Laser Science, DESY and University of Hamburg, Notkestraße 85, \\ D-22607Hamburg, Germany \\ cristian.manzoni@polimi.it
}

\begin{abstract}
We report on coherent synthesis of two ultra-broadband optical parametric amplifiers, resulting in octave-spanning $(500-1000 \mathrm{~nm})$ spectra supporting nearly single-cycle sub-4-fs pulse duration. Synthesized pulse timing is locked to sub-300-as by a balanced cross-correlator.
\end{abstract}

OCIS codes: (320.7110) Ultrafast nonlinear optics; (320.7160) Ultrafast technology; (190.4975) Parametric processes.

\section{Introduction}

The generation of sub-single-cycle, carrier-envelope phase (CEP) stable light pulses is one of the frontiers of ultrafast optics, promising to break tremendous new ground in the control of strong-field processes such as highharmonic and attosecond pulse generation [1]. These pulses allow arbitrary shaping of the strong electric-field waveform for steering ionized electron wavepackets and precise control of tunneling and multiphoton ionization events. The two key ingredients for sub-single-cycle pulse generation are ultrabroad bandwidths, exceeding one octave, and accurate control of the spectral phase, so as to obtain nearly transform-limited (TL) pulsewidths. These requirements are very challenging to satisfy with a single laser source, and thus intense research activity is devoted to the coherent synthesis of pulses generated by separate sources. Coherent addition of two pulse trains derived from the same fiber laser, and spectrally broadened by self-phase-modulation (SPM) in an optical fiber, resulted in the first demonstration of an isolated single-cycle optical pulse [2], although with low (nJ-level) energy. SPM in a hollow-core fiber, followed by dispersion management of different portions of the ultra-broadband spectrum and coherent superposition of the compressed sub-pulses, has recently allowed the generation of sub-cycle light pulses with hundreds of $\mu \mathrm{Js}$ energy [3]. However, due to the high pressures used in the hollow-fiber compressor, such pulses have strongly modulated spectra and further energy scaling is limited by the handling capabilities of the fiber.

Coherent combination of ultra-broadband optical parametric amplifiers (OPAs) or optical parametric chirped pulse amplifiers (OPCPAs) is an alternative promising route to sub-single-cycle pulse synthesis, with the advantages of smooth spectra and straightforward energy scaling capabilities. A first milestone in this direction was the recent demonstration of the synthesis of two OPCPAs, at $800 \mathrm{~nm}$ and $2 \mu \mathrm{m}$ respectively, which has enabled sub-cycle waveform shaping over a two-octave-spanning spectrum [4].

Here we demonstrate coherent synthesis of two ultra-broadband OPAs pumped by a Ti:sapphire laser and seeded by the same white-light continuum (WLC). OPAs are powerful tools for the generation of tunable fewoptical-cycle light pulses [5]. Broadband gain in an OPA is achieved when the group velocities of signal and idler are matched [5]; this condition is satisfied either in the case of a degenerate OPA (DOPA) with type I phase matching, or in the non-collinear OPA (NOPA), in which the idler group velocity is projected along the signal propagation direction to match that of the signal. Using these concepts, a variety of broadband OPA schemes, pumped by either the fundamental frequency (FF) or the second harmonic (SH) of Ti:Sapphire, have been demonstrated. In particular, 5-fs visible pulses $(500-700 \mathrm{~nm})$ are routinely generated by SH-pumped NOPA [6], and sub-7-fs pulses have been produced in the $650-950 \mathrm{~nm}$ spectral region by the SH-pumped DOPA [7]. Here we demonstrate coherent synthesis of the NOPA and the DOPA, resulting in octave-spanning (500-1000 nm) spectra supporting nearly single-cycle sub-4-fs-pulses.

\section{Experimental setup and results}

The experimental setup for coherent synthesis of OPAs, summarized in the block diagram of Fig. 1, combines (i) the generation of a broadband WLC seed with (ii) two broadband OPAs seeded by distinct portions of the WLC and (iii) coherent combination of their outputs in order to synthesize high energy (sub)single-cycle pulses. The system is 
powered by $550-\mu \mathrm{J}$ pulses from an amplified, non CEP-stabilized Ti:sapphire laser, providing 100 -fs pulses at 800 $\mathrm{nm}$ wavelength and $1-\mathrm{kHz}$ repetition rate. Since the WLC presents a highly structured intensity and phase profile around the driving pulse wavelength, it is not possible to use the WLC directly generated by the FF in order to seed the DOPA, and an additional stage is necessary to shift the WLC driving wavelength to the infrared, thus generating a well-behaved WLC around $800 \mathrm{~nm}$. The two-stage near-IR OPA is pumped by the FF of Ti:sapphire and seeded by the WLC generated in a sapphire plate. When pumped by $250-\mu \mathrm{J}$ pulses, this OPA provides a total signal + idler energy of $35 \mu \mathrm{J}$. An additional benefit of the near-IR OPA is the possibility to produce idler pulses with passively stabilized CEP, exploiting the difference frequency generation process that occurs between pump and signal sharing the same CEP [8]. Both the signal and the CEP-stable idler can be used to generate a broadband, stable and spectrally well-behaved WLC, either in a 3-mm thick sapphire plate or in a 4-mm thick YAG plate, respectively.

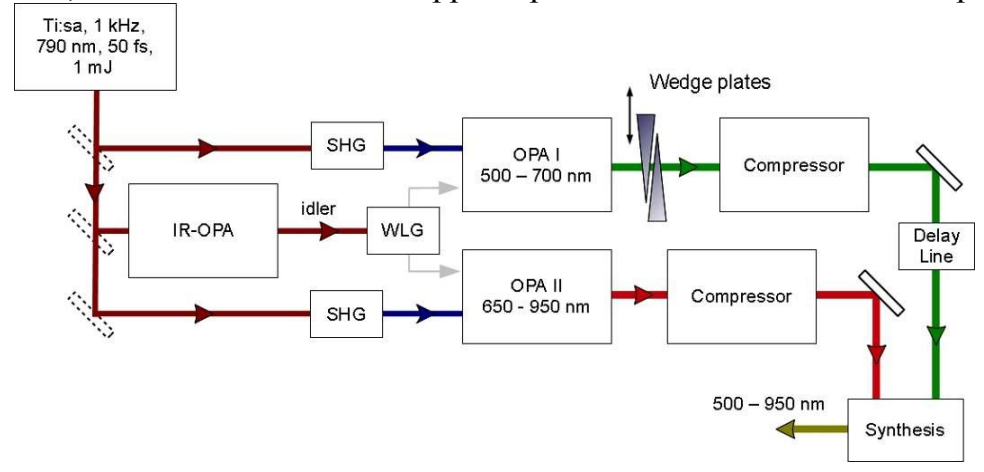

Fig. 1: setup for separate amplification, compression and coherent combination of visible and near-infrared pulses. SHG: second harmonic generation; WLG: white light generation.

The short-wavelength portion of this seed ranges from 500 to $1000 \mathrm{~nm}$, and covers without gaps the amplification range of the subsequent visible NOPA and SH-pumped DOPA (see Fig 2). For this proof-of-principle demonstration, we tuned the near-IR-OPA to $1.3 \mu \mathrm{m}$ and used its signal beam for WLC generation in sapphire. The seed is then split by a neutral-density ultrathin beam-splitter, and the two replicas are amplified by the visible NOPA and by the DOPA. Both OPAs are pumped by the SH from the remaining $300-\mu \mathrm{J}$ FF pulses; thick SH crystals were used to narrow the bandwidth of the SH pulse, and to facilitate its temporal overlap with the chirped WLC. Each of the two OPAs provides pulses with an energy of 1-2 $\mu \mathrm{J}$. To ensure good spatial overlap of the collimated beams, the OPAs employ the same focal lengths and propagate the beams for the same distances. Compression of the two OPA outputs is achieved by visible and IR chirped mirrors, which provide pulsewidths close to the TL values ( $\approx 6$ fs for the visible NOPA and $\approx 8$ fs for the DOPA).

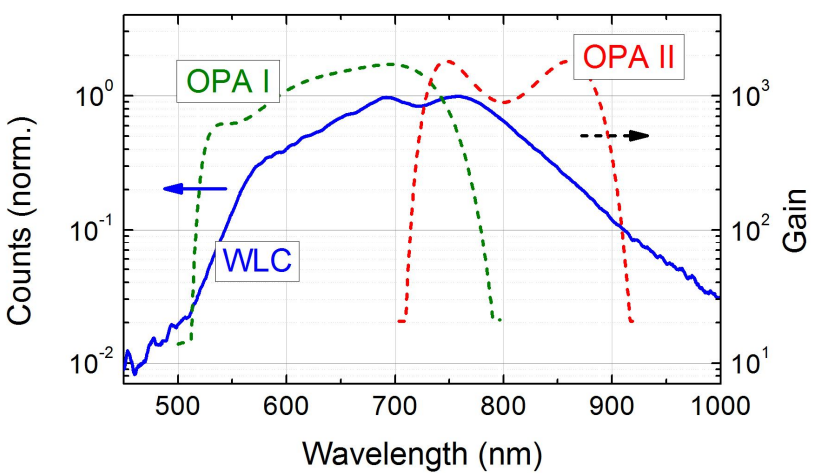

(a) (b)

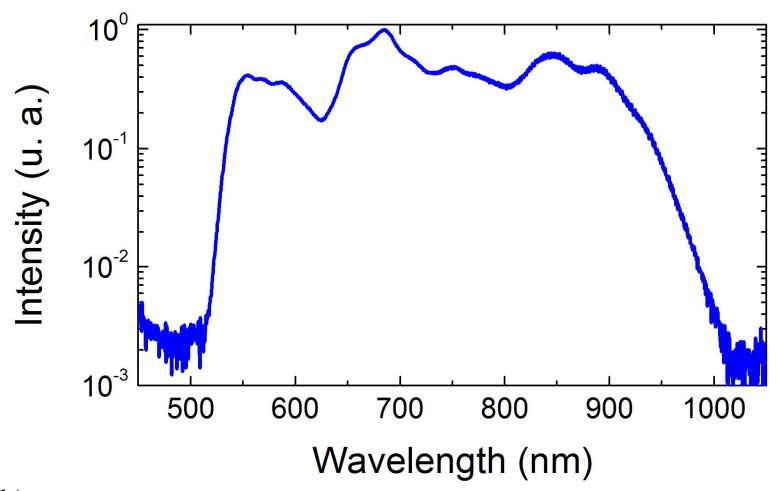

Fig.2: (a) Spectrum of the gap-free WLC(blue solid line) generated in the sapphire plate, compared to the gain of the visible (dashed green line) and infrared (dashed red line) OPAs; (b) Spectrum of the synthesized pulses, supporting 3.5 fs pulse duration.

The two amplified pulses are then synchronized by a delay line equipped with a piezoelectric actuator, and collinearly combined by a second ultrathin beam-splitter. A gap-free spectrum arising from the combination of the two pulses is shown in Fig. 2(b); it ranges from 520 to $980 \mathrm{~nm}$ with very good spectral uniformity (variations with frequency lower than $3 \mathrm{~dB}$ ) and supports a transform-limited 3.5 fs pulse duration. The last step of the pulse 
synthesis is the coherent combination of the two pulses, which calls for careful control of their relative delay and CEP. The relative CEP can be finely tuned by a wedge pair inserted in the path of one of the beams. The relative delay of the pulses was detected by a balanced optical cross-correlator (BOC) [9], which consists of two nearly identical cross-correlators using 1-mm-thick BBO crystals, phase matched for sum-frequency generation of 600-nm light from the NOPA and 800 -nm light from the DOPA. The BOC allows attosecond-precision relative timing measurement thanks to the capability of the balanced detection scheme to cancel the amplitude noise. The signal from the BOC was low-pass filtered and fed to a PID controller driving the piezo translator and the delay line. The closed loop allowed suppression of relative delay fluctuations between the two pulses to below 300 -as over a measurement time of 10 minutes, corresponding to less than 1/8 optical cycle (Fig. 3); this is compared to the open loop rms fluctuations of $\approx 3 \mathrm{fs}$ of the unlocked pulses. The fringe pattern at the spectral overlap between the visible and IR light given in panel (b) confirms the high interferometric stability achieved with the closed loop. The last remaining experimental challenge will be full temporal characterization of the synthesized ultra-broadband pulse, which will be performed by two-dimensional spectral-shearing interferometry [10].
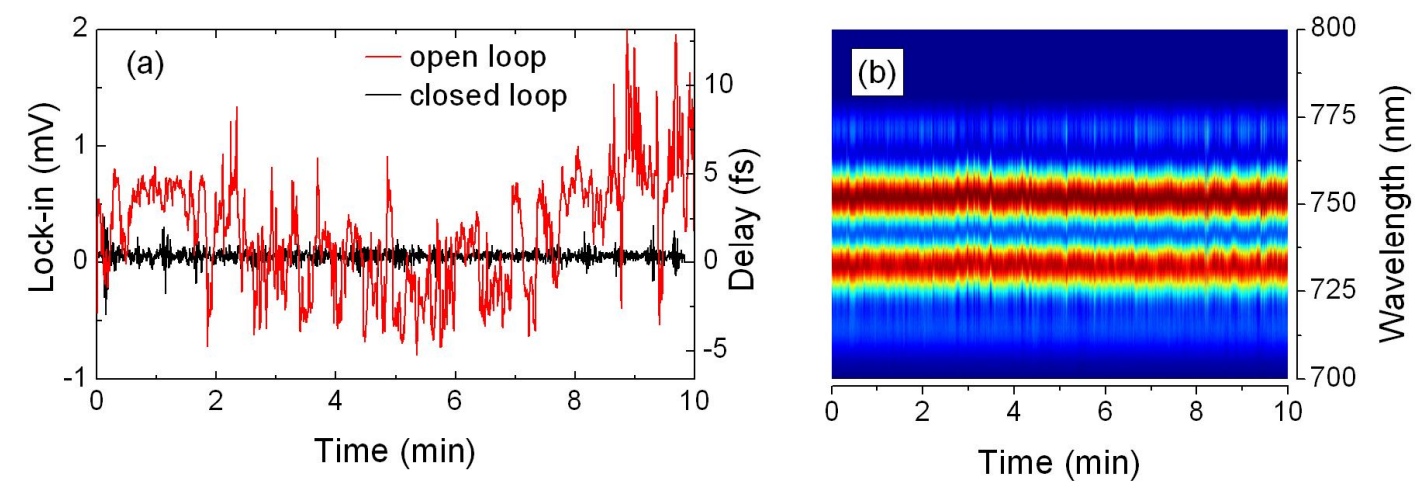

Fig.3: (a) Signal from the BCC and corresponding pulse delay. Red line: free-run operation of the system; black line: pulse delay is locked by the feedback. (b) Interference fringes at the overlap between the visible and IR spectra recorded when locking is active.

\section{Conclusions}

We have demonstrated coherent synthesis of pulses from two ultra-broadband OPAs, pumped by the SH of Ti:sapphire; the combined spectrum extends from 520 to $980 \mathrm{~nm}$, corresponding to 3.5 -fs TL pulses. The relative timing of the two OPAs was locked to less than 300 as thanks to a feedback loop based on a BOC. The possibility to seed the OPAs with CEP-stable light allows the generation of nearly single-cycle pulses with fully controlled electric field. This is a proof-of-principle experiment with limited available pump energy, however scaling of the energy of both OPAs to the mJ-level by addition of power amplification stages should be straightforward. Furthermore, further wavelength multiplexing by the coherent addition of other ultra-broadband OPAs [11] should allow the generation of high-quality sub-cycle pulses.

\section{References}

[1] E. Goulielmakis et al., "Single-Cycle Nonlinear Optics," Science, 320, 1614 (2008).

[2] G. Krauss et al., "Synthesis of a single cycle of light with compact erbium-doped fibre technology" Nature Photonics 4, 33 (2010).

[3] A. Wirth et al., "Synthesized Light Transients", Science, in press (2011).

[4] S.W. Huang et al., "High-energy pulse synthesis with sub-cycle waveform control for strong-field physics", Nature Photonics 5, 475 (2011).

[5] G. Cerullo and S. De Silvestri, "Ultrafast optical parametric amplifiers," Rev. Sci. Instr. 74, 1 (2003).

[6] A. Baltuška, T. Fuji, and T. Kobayashi, "Visible pulse compression to 4 fs by optical parametric amplification and programmable dispersion control", Opt. Lett. 27, 306 (2002).

[7] A. M. Siddiqui, G. Cirmi, D. Brida, F. X. Kärtner, and G. Cerullo, "Generation of $<7$ fs pulses at $800 \mathrm{~nm}$ from a blue-pumped optical parametric amplifier at degeneracy," Opt. Lett. 34, 3592 (2009).

[8] A. Baltuška, T. Fuji, and T. Kobayashi, "Controlling the carrier-envelope phase of ultrashort light pulses with optical parametric amplifiers", Phys. Rev. Lett. 88, 133901 (2002).

[9] T.R. Schibli et al. "Attosecond active synchronization of passively mode-locked lasers by balanced cross correlation", Opt. Lett. 28, 947 (2003).

[10] J.R. Birge, H.M. Crespo, and F.X. Kärtner, "Theory and design of two-dimensional spectral shearing interferometry for few-cycle pulse measurement”, J. Opt. Soc. Am. B 27, 1165 (2010).

[11] D. Brida, C. Manzoni, G. Cirmi, M. Marangoni, S. Bonora, P. Villoresi, S. De Silvestri and G. Cerullo, "Few-optical-cycle pulses tunable from the visible to the mid-infrared by optical parametric amplifiers", J. Opt. 12, 013001 (2010). 